Acta Crystallographica Section E

Structure Reports

Online

ISSN 1600-5368

\section{N. David Karis, ${ }^{\text {a }}$ Wendy A. Loughlin, ${ }^{b}$ Ian D. Jenkins ${ }^{a}$ and Peter C. Healy ${ }^{b_{*}}$}

${ }^{a}$ Natural Product Discovery and Eskitis Institute, Griffith University, Nathan, Brisbane 4111, Australia, and ${ }^{\mathbf{b}}$ School of Science and Eskitis Institute, Griffith University, Nathan, Brisbane 4111, Australia

Correspondence e-mail: p.healy@griffith.edu.au

\section{Key indicators}

Single-crystal X-ray study

$T=295 \mathrm{~K}$

Mean $\sigma(\mathrm{C}-\mathrm{C})=0.004 \AA$

$R$ factor $=0.045$

$w R$ factor $=0.143$

Data-to-parameter ratio $=12.8$

For details of how these key indicators were automatically derived from the article, see http://journals.iucr.org/e.

\title{
Ethyl (3-nitro-2-oxo-1,2-dihydropyridin-1-yl)acetate
}

The title compound, $\mathrm{C}_{9} \mathrm{H}_{10} \mathrm{~N}_{2} \mathrm{O}_{5}$, crystallizes with two crystallographically independent molecules in the asymmetric unit. In the crystal structure, the nitropyridone rings are connected by weak $\mathrm{C}-\mathrm{H} \cdots \mathrm{O}$ interactions, forming sheet-like arrays, which are in turn linked by $\mathrm{C}-\mathrm{H} \cdots \pi$ and $\pi-\pi$ interactions between the nitropyridone rings on one side, and by $\mathrm{C}-$ $\mathrm{H} \cdots \mathrm{O}$ and van der Waals interactions between the ester groups on the other.

\section{Comment}

Pyridones can act as a P3 to P2 conformational restraint in the design of inhibitors of serine protease enzymes (Loughlin et $a l ., 2004)$. This is often facilitated by the presence of a 3-amino group, which acts as a hydrogen-bonding site. Typically, 3nitropyrid-2-ones (I) and (II) are used as synthetic precursors to 3-aminopyrid-2-ones (Breslin et al., 2003; Huang et al., 2003; Reiner et al., 2002; Warner et al., 1994). Compound (II) lacks the hydrogen-bonding site at the 3 position and solid-state structures for 3-nitropyridones (without C4 to C6 substituents) are restricted to a report of the complex of (I) with 2amino-5-nitropyridine (Velikova et al., 1997). Here, we report the first solid-state structure of the title compound, (II). Compound (II) was prepared by $N$-alkylation of (I) with sodium hydride and ethyl bromoacetate, as reported elsewhere (Warner et al., 1994; Breslin et al., 2003).<smiles>O=c1[nH]cccc1[N+](=O)[O-]</smiles>

(I)

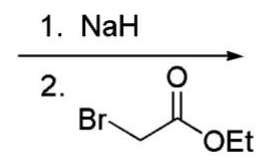

(II)

The crystal structure of (II) contains two crystallographically independent molecules in the asymmetric unit (Figs. 1 and 2). Relevant bond lengths and angles are listed in Table 1 and are in accord with those reported for 3-nitro-2pyridone (Velikova et al., 1997). The nitro groups are twisted slightly out of the plane of the pyridone ring, with torsion angles $\mathrm{O} 32-\mathrm{N} 3-\mathrm{C} 3-\mathrm{C} 2=-18.5(4)^{\circ}$ and $\mathrm{O} 132-\mathrm{N} 13-$ $\mathrm{C} 13-\mathrm{C} 12=22.4(4)^{\circ}$.

In the crystal structure, the nitropyridone rings are connected by weak $\mathrm{C}-\mathrm{H} \cdots \mathrm{O}$ interactions between the aromatic and aliphatic $\mathrm{H}$ atoms and the carbonyl and nitro group $\mathrm{O}$ atoms, forming sheet-like arrays in the $b c$ plane. These sheets are in turn linked together by $\mathrm{C}-\mathrm{H} \cdots \pi$ and $\pi-\pi$ interactions between the nitropyridone rings on one side, and by $\mathrm{C}-\mathrm{H} \cdots \mathrm{O}$ and van der Waals interactions between the ester groups on the other (Fig. 2).
Received 22 May 2006 Accepted 30 May 2006 


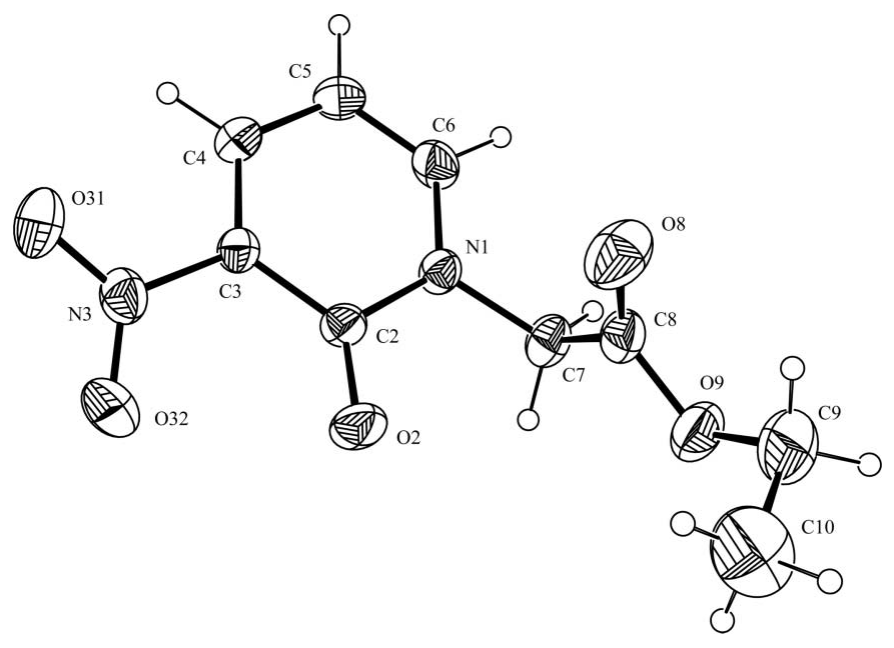

Figure 1

A view of one of the two independent molecules of (II), with the atomnumbering scheme. Displacement ellipsoids are drawn at the $30 \%$ probability level.

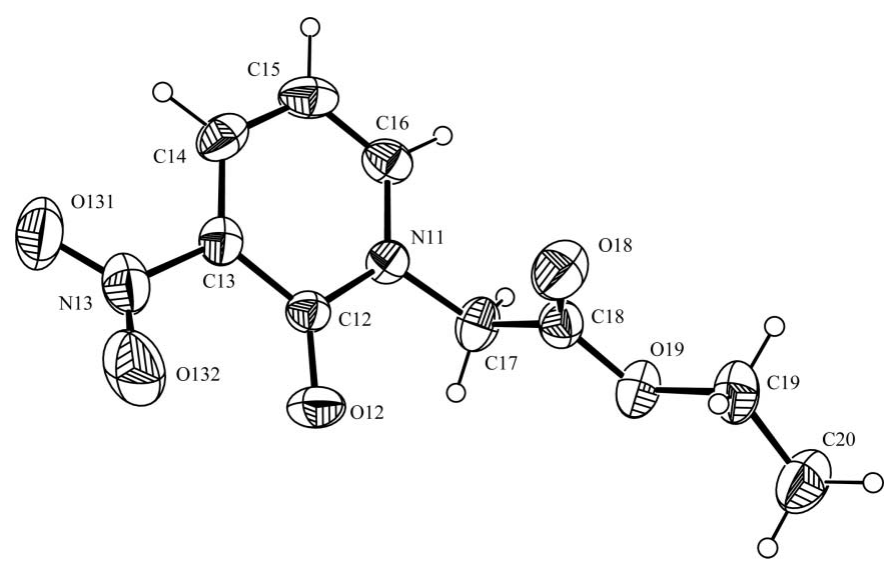

Figure 2

A view of the second of the two independent molecules of (II), with the atom-numbering scheme. Displacement ellipsoids are drawn at the $30 \%$ probability level.

\section{Experimental}

Sodium hydride $(1.38 \mathrm{~g}, 57.8 \mathrm{mmol})$ was added in portions over a period of $30 \mathrm{~min}$ to compound (I) $(6.75 \mathrm{~g}, 48.2 \mathrm{mmol})$ in dry tetrahydrofuran $(100 \mathrm{ml})$. The resulting suspension was stirred for $30 \mathrm{~min}$. Ethyl bromoacetate $(5.86 \mathrm{ml}, 53.0 \mathrm{mmol})$ was added dropwise over a period of $30 \mathrm{~min}$. The resulting yellow suspension was heated to $328 \mathrm{~K}$ under nitrogen for $24 \mathrm{~h}$. The red reaction mixture was filtered and the solid thoroughly washed with ethyl acetate. The filtrate was concentrated under reduced pressure and the resulting red oil purified by silica-gel column chromatography (ethyl acetate-dichloromethane gradient from $0-40 \%$ ethyl acetate, with $0.4 \%$ triethylamine). Pale-yellow crystals of compound (II) (m.p. 327$329 \mathrm{~K}$; yield $10.7 \mathrm{~g}, 98 \%$ ) were isolated by slow evaporation of an ethyl acetate solution of (II). Analysis, found: C 47.82, $\mathrm{H} \mathrm{4.44,}$ N 12.34\%; calculated for $\mathrm{C}_{9} \mathrm{H}_{10} \mathrm{~N}_{2} \mathrm{O}_{5}$ : C 47.79, $\mathrm{H} 4.46, \mathrm{~N} 12.38 \%$. Spectroscopic analysis: ${ }^{1} \mathrm{H}$ NMR (400 MHz, $\mathrm{CDCl}_{3}$, $\delta$, p.p.m.): 1.293 $\left(3 \mathrm{H}, t, J=7.2 \mathrm{~Hz}, \mathrm{CH}_{3}\right), 4.251\left(2 \mathrm{H}, q, J=7.2 \mathrm{~Hz}, \mathrm{CH}_{2}\right), 4.769(2 \mathrm{H}, s$, $\left.\mathrm{NCH}_{2}\right), 6.380(1 \mathrm{H}, d d, J=7.6$ and $6.8 \mathrm{~Hz}, \mathrm{H} 5), 7.712(1 \mathrm{H}, d d, J=6.8$ and $2.0 \mathrm{~Hz}, \mathrm{H} 6), 8.387(1 \mathrm{H}, d d, J=7.6$ and $2.0 \mathrm{~Hz}, \mathrm{H} 4) ;{ }^{13} \mathrm{C} \mathrm{NMR}$ (100 MHz, $\mathrm{CDCl}_{3}$, $\delta$, p.p.m.): $14.3\left(\mathrm{CH}_{3}\right), 51.4\left(\mathrm{NCH}_{2}\right), 62.6\left(\mathrm{CH}_{2}\right)$, 103.8 (C5), 138.9 (C3), 139.7 (C4), 145.2 (C6), 154.5 (C2), 166.8 (CO); MS (ES $): 248.8\left(\mathrm{MNa}^{+}, 60 \%\right) 226.8\left(\mathrm{MH}^{+}, 70 \%\right) 180.7\left(\mathrm{MH}^{+}-\mathrm{NO}_{2}\right.$, $100 \%)$.

\section{Crystal data}

$\mathrm{C}_{9} \mathrm{H}_{10} \mathrm{~N}_{2} \mathrm{O}_{5}$

$M_{r}=226.19$

Monoclinic, $P 2_{1} / c$

$a=20.175(4) \AA$

$b=8.333(3) \AA$

$c=12.729(4) \AA$

$\beta=100.49(2)^{\circ}$

$V=2104.2(11) \AA^{3}$

$$
\begin{aligned}
& Z=8 \\
& D_{x}=1.428 \mathrm{Mg} \mathrm{m}^{-3} \\
& \text { Mo } K \alpha \text { radiation } \\
& \mu=0.12 \mathrm{~mm}^{-1} \\
& T=295 \mathrm{~K} \\
& \text { Prism, yellow } \\
& 0.40 \times 0.30 \times 0.20 \mathrm{~mm}
\end{aligned}
$$

\section{Data collection}

Rigaku AFC-7 $R$ diffractometer $\omega / 2 \theta$ scans

Absorption correction: none

4183 measured reflections

3689 independent reflections

1988 reflections with $I>2 \sigma(I)$

$$
\begin{aligned}
& R_{\text {int }}=0.027 \\
& \theta_{\max }=25.0^{\circ} \\
& 3 \text { standard reflections } \\
& \text { every } 150 \text { reflections } \\
& \text { intensity decay: } 1.1 \%
\end{aligned}
$$

\begin{tabular}{|c|c|c|c|}
\hline $\mathrm{O} 2-\mathrm{C} 2$ & $1.220(3)$ & O131-N13 & $1.228(4)$ \\
\hline $\mathrm{O} 8-\mathrm{C} 8$ & $1.188(4)$ & $\mathrm{O} 132-\mathrm{N} 13$ & $1.212(5)$ \\
\hline $\mathrm{O} 9-\mathrm{C} 8$ & $1.319(3)$ & $\mathrm{N} 1-\mathrm{C} 2$ & $1.408(3)$ \\
\hline $\mathrm{O} 9-\mathrm{C} 9$ & $1.448(4)$ & $\mathrm{N} 1-\mathrm{C} 6$ & $1.347(3)$ \\
\hline $\mathrm{O} 31-\mathrm{N} 3$ & $1.218(4)$ & $\mathrm{N} 1-\mathrm{C} 7$ & $1.463(3)$ \\
\hline $\mathrm{O} 32-\mathrm{N} 3$ & $1.226(3)$ & $\mathrm{N} 3-\mathrm{C} 3$ & $1.446(4)$ \\
\hline $\mathrm{O} 12-\mathrm{C} 12$ & $1.221(3)$ & $\mathrm{N} 11-\mathrm{C} 12$ & $1.396(3)$ \\
\hline $\mathrm{O} 18-\mathrm{C} 18$ & $1.196(4)$ & $\mathrm{N} 11-\mathrm{C} 16$ & $1.342(4)$ \\
\hline $\mathrm{O} 19-\mathrm{C} 19$ & $1.471(4)$ & $\mathrm{N} 11-\mathrm{C} 17$ & $1.464(4)$ \\
\hline $\mathrm{O} 19-\mathrm{C} 18$ & $1.328(4)$ & $\mathrm{N} 13-\mathrm{C} 13$ & $1.458(4)$ \\
\hline $\mathrm{C} 8-\mathrm{O} 9-\mathrm{C} 9$ & $118.6(2)$ & $\mathrm{N} 3-\mathrm{C} 3-\mathrm{C} 4$ & $117.6(2)$ \\
\hline $\mathrm{C} 18-\mathrm{O} 19-\mathrm{C} 19$ & $116.4(2)$ & $\mathrm{N} 1-\mathrm{C} 6-\mathrm{C} 5$ & $121.7(2)$ \\
\hline $\mathrm{C} 2-\mathrm{N} 1-\mathrm{C} 6$ & $123.5(2)$ & $\mathrm{N} 1-\mathrm{C} 7-\mathrm{C} 8$ & $111.0(2)$ \\
\hline $\mathrm{C} 6-\mathrm{N} 1-\mathrm{C} 7$ & 120.73 (19) & $\mathrm{O} 8-\mathrm{C} 8-\mathrm{C} 7$ & $124.9(3)$ \\
\hline $\mathrm{C} 2-\mathrm{N} 1-\mathrm{C} 7$ & 115.72 (19) & $\mathrm{O} 9-\mathrm{C} 8-\mathrm{C} 7$ & $110.4(2)$ \\
\hline $\mathrm{O} 31-\mathrm{N} 3-\mathrm{O} 32$ & $122.3(3)$ & $\mathrm{O} 8-\mathrm{C} 8-\mathrm{O} 9$ & 124.7 (3) \\
\hline $\mathrm{O} 32-\mathrm{N} 3-\mathrm{C} 3$ & $119.2(2)$ & $\mathrm{O} 9-\mathrm{C} 9-\mathrm{C} 10$ & $111.1(3)$ \\
\hline $\mathrm{O} 31-\mathrm{N} 3-\mathrm{C} 3$ & $118.6(2)$ & $\mathrm{O} 12-\mathrm{C} 12-\mathrm{N} 11$ & $118.9(2)$ \\
\hline $\mathrm{C} 12-\mathrm{N} 11-\mathrm{C} 17$ & $116.8(2)$ & $\mathrm{O} 12-\mathrm{C} 12-\mathrm{C} 13$ & $128.1(2)$ \\
\hline $\mathrm{C} 16-\mathrm{N} 11-\mathrm{C} 17$ & $119.0(3)$ & $\mathrm{N} 11-\mathrm{C} 12-\mathrm{C} 13$ & $112.9(2)$ \\
\hline $\mathrm{C} 12-\mathrm{N} 11-\mathrm{C} 16$ & $124.1(3)$ & $\mathrm{N} 13-\mathrm{C} 13-\mathrm{C} 12$ & $118.5(2)$ \\
\hline $\mathrm{O} 132-\mathrm{N} 13-\mathrm{C} 13$ & $119.7(3)$ & $\mathrm{N} 13-\mathrm{C} 13-\mathrm{C} 14$ & $119.1(3)$ \\
\hline $\mathrm{O} 131-\mathrm{N} 13-\mathrm{O} 132$ & $123.8(4)$ & $\mathrm{N} 11-\mathrm{C} 16-\mathrm{C} 15$ & $121.6(3)$ \\
\hline $\mathrm{O} 131-\mathrm{N} 13-\mathrm{C} 13$ & $116.5(3)$ & $\mathrm{N} 11-\mathrm{C} 17-\mathrm{C} 18$ & $110.8(3)$ \\
\hline $\mathrm{O} 2-\mathrm{C} 2-\mathrm{N} 1$ & $118.0(2)$ & $\mathrm{O} 18-\mathrm{C} 18-\mathrm{O} 19$ & $124.7(3)$ \\
\hline $\mathrm{O} 2-\mathrm{C} 2-\mathrm{C} 3$ & $129.3(2)$ & $\mathrm{O} 18-\mathrm{C} 18-\mathrm{C} 17$ & $124.8(3)$ \\
\hline $\mathrm{N} 1-\mathrm{C} 2-\mathrm{C} 3$ & $112.7(2)$ & $\mathrm{O} 19-\mathrm{C} 18-\mathrm{C} 17$ & $110.5(3)$ \\
\hline $\mathrm{N} 3-\mathrm{C} 3-\mathrm{C} 2$ & $119.1(2)$ & $\mathrm{O} 19-\mathrm{C} 19-\mathrm{C} 20$ & $108.2(3)$ \\
\hline
\end{tabular}

\section{Refinement}

Refinement on $F^{2}$

$R\left[F^{2}>2 \sigma\left(F^{2}\right)\right]=0.045$

$w R\left(F^{2}\right)=0.143$

$S=1.03$

3689 reflections

289 parameters

$\mathrm{H}$-atom parameters constrained

$$
\begin{aligned}
& w=1 /\left[\sigma^{2}\left(F_{\mathrm{o}}{ }^{2}\right)+(0.058 P)^{2}\right. \\
& \quad+0.4402 P] \\
& \quad \text { where } P=\left(F_{\mathrm{o}}{ }^{2}+2 F_{\mathrm{c}}{ }^{2}\right) / 3 \\
& (\Delta / \sigma)_{\max }=0.010 \\
& \Delta \rho_{\max }=0.17 \mathrm{e} \AA^{-3} \\
& \Delta \rho_{\min }=-0.18 \mathrm{e}^{-3}
\end{aligned}
$$

Table 1

Selected geometric parameters $\left(\AA{ }^{\circ}\right)$. 
Table 2

Hydrogen-bond geometry $\left(\AA,^{\circ}\right)$.

\begin{tabular}{lllll}
\hline$D-\mathrm{H} \cdots A$ & $D-\mathrm{H}$ & $\mathrm{H} \cdots A$ & $D \cdots A$ & $D-\mathrm{H} \cdots A$ \\
\hline $\mathrm{C} 4-\mathrm{H} 4 \cdots \mathrm{O} 2^{\mathrm{i}}$ & 0.95 & 2.24 & $3.117(3)$ & 154 \\
$\mathrm{C} 5-\mathrm{H} 5 \cdots \mathrm{O} 32^{\mathrm{i}}$ & 0.95 & 2.64 & $3.457(4)$ & 145 \\
$\mathrm{C} 16-\mathrm{H} 16 \cdots \mathrm{O} 12^{\mathrm{i}}$ & 0.95 & 2.17 & $3.049(4)$ & 153 \\
$\mathrm{C} 17-\mathrm{H} 172 \cdots \mathrm{O} 132^{\mathrm{i}}$ & 0.95 & 2.67 & $3.536(4)$ & 152 \\
$\mathrm{C} 9-\mathrm{H} 91 \cdots$ O8 $^{\mathrm{ii}}$ & 0.95 & 2.57 & $3.517(5)$ & 173 \\
\hline
\end{tabular}

Symmetry codes: (i) $x,-y-\frac{1}{2}, z+\frac{1}{2}$; (ii) $-x+1, y+\frac{1}{2},-z+\frac{3}{2}$.

$\mathrm{H}$ atoms were constrained as riding atoms, with $\mathrm{C}-\mathrm{H}=0.95 \AA$, and with $U_{\text {iso }}(\mathrm{H})=1.2 U_{\text {eq }}(\mathrm{C})$.

Data collection: MSC/AFC7 Diffractometer Control Software (Molecular Structure Corporation, 1999); cell refinement: $M S C /$ AFC7 Diffractometer Control Software; data reduction: TEXSAN for Windows (Molecular Structure Corporation, 2001); program(s) used to solve structure: TEXSAN for Windows; program(s) used to refine structure: TEXSAN for Windows and SHELXL97 (Sheldrick, 1997); molecular graphics: ORTEP-3 (Farrugia, 1997); software used to prepare material for publication: TEXSAN for Windows and PLATON (Spek, 2003).

The authors acknowledge financial support of this work by Griffith University, by Eskitis Institute of Cell and Molecular Therapies, Griffith University, and by Natural Product Discovery, Griffith University.

\section{References}

Breslin, M. J., Duggan, M. E., Halczenko, W., Fernandez-Metzler, C., Hunt, C. A., Leu, C.-T., Merkle, K. M., Naylor-Olsen, A. M., Prueksaritanont, T., Stump, G., Wallace, A., Rodan, S. B. \& Hutchinson, J. H. (2003). Bioorg. Med. Chem. Lett. 13, 1809-1812.

Farrugia, L. J. (1997). J. Appl. Cryst. 30, 565.

Huang, W., Naughton, M. A., Yang, H., Su, T., Dam, S., Wong, P. W., Arfsten, A., Edwards, S., Sinha, U., Hollenbach, S., Scarborough, R. M. \& Zhu, B.-Y. (2003). Bioorg. Med. Chem. Lett. 13, 723-728.

Loughlin, W. A., Tyndall, J. D. A., Glenn, M. P. \& Fairlie, D. P. (2004). Chem. Rev. 104, 6085-6117 and references therein.

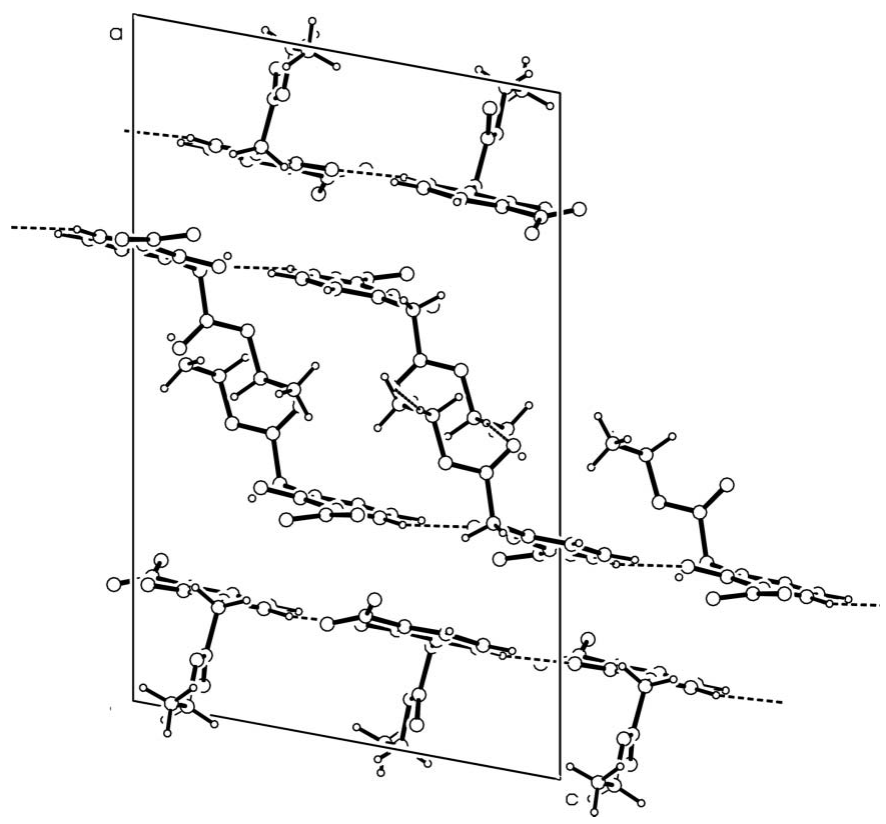

Figure 3

The crystal packing of (I), viewed down the $b$ axis. Dashed lines indicate hydrogen bonds.

Molecular Structure Corporation (1999). MSC/AFC7 Diffractometer Control Software. Version 1.02 for Windows. MSC, 9009 New Trails Drive, The Woodlands, TX 77381, USA.

Molecular Structure Corporation (2001). TEXSAN for Windows. Version 1.06. MSC, 9009 New Trails Drive, The Woodlands, TX 77381, USA.

Reiner, J. E., Siev, D. V., Araldi, G.-L., Cui, J. J., Ho, J. Z., Reddy, K. M., Mamedova, L., Vu, P. H., Lee, K.-S. S., Minami, N. K., Gibson, T. S., Anderson, S. M., Bradbury, A. E., Nolan, T. G. \& Semple, J. E. (2002). Bioorg. Med. Chem. Lett. 12, 1203-1208.

Sheldrick, G. M. (1997). SHELXL97. University of Göttingen, Germany. Spek, A. L. (2003). J. Appl. Cryst. 36, 7-13.

Velikova, V., Kossev, K. \& Angelova, O. (1997). Acta Cryst. C53, 1270-1273.

Warner, P., Green, R. C., Gomes, B. \& Strimpler, A. M. (1994). J. Med. Chem. 37, 3090-3099. 